KEK preprint 95-179

OCU-HEP 95-02

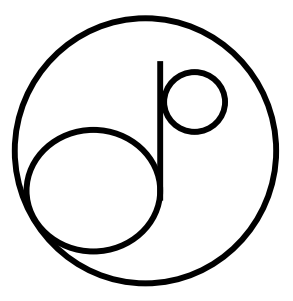

NWU-HEP 95-03

TUAT-HEP 95-01

DPNU-95-22

PU-95-697

\title{
Observation of Highly Virtual Photon-Photon Collisions to Hadrons at TRISTAN*
}

\author{
$\underline{\text { R.Enomoto }^{(1) \dagger}}$, K.Abe $^{(2)}$, T.Abe $^{(2)}$, I.Adachi $^{(1)}$, K.Adachi $^{(3)}$, M.Aoki $^{(4)}$, M.Aoki $^{(2)}$,

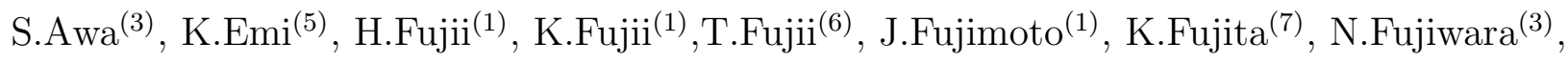

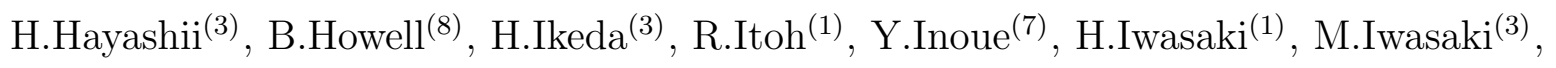

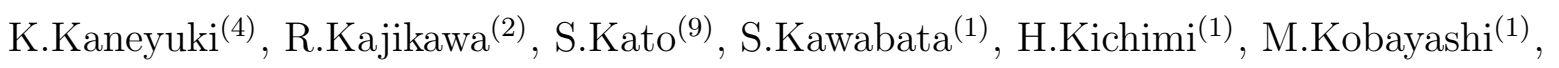 \\ D.Koltick $^{(8)}$, I.Levine ${ }^{(8)}$, S.Minami ${ }^{(4)}$, K.Miyabayashi ${ }^{(3)}$, A.Miyamoto $^{(1)}$ K.Nagai $^{(10) \ddagger}$,

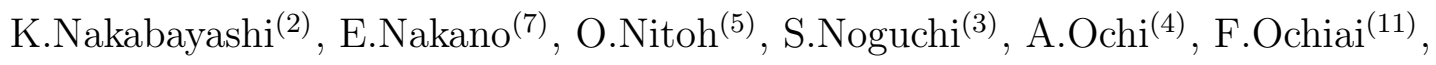

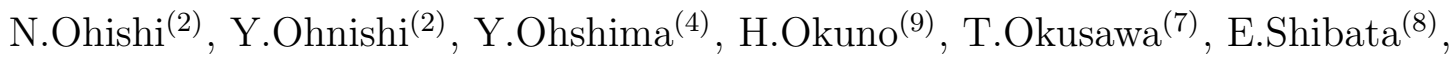 \\ T.Shinohara ${ }^{(5)}$, A.Sugiyama ${ }^{(2)}$, S.Suzuki ${ }^{(2)}$, S.Suzuki ${ }^{(4)}$, K.Takahashi ${ }^{(5)}$, T.Takahashi ${ }^{(7)}$,

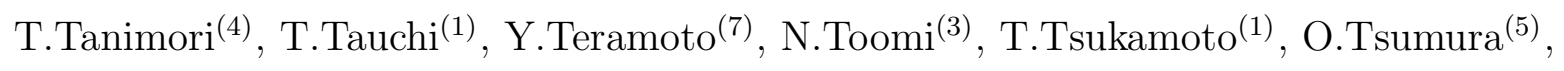 \\ S.Uno $^{(1)}$, T.Watanabe ${ }^{(4)}$, Y.Watanabe ${ }^{(4)}$, A.Yamaguchi ${ }^{(3)}$, A.Yamamoto ${ }^{(1)}$, and \\ M.Yamauchi ${ }^{(1)}$ \\ (TOPAZ Collaboration) \\ (1) KEK, National Laboratory for High Energy Physics, Ibaraki-ken 305, Japan
}

\footnotetext{
*to be published in Phys. Lett. B

†internet address: enomoto@kekvax.kek.jp.

†Present address; Dept. of Particle Phys., The weizmann Inst. of Sci., Rehovot 76100, Israel
} 
${ }^{(2)}$ Department of Physics, Nagoya University, Nagoya 464, Japan

(3) Department of Physics, Nara Women's University, Nara 630, Japan

(4) Department of Physics, Tokyo Institute of Technology, Tokyo 152, Japan

(5) Dept. of Appl. Phys., Tokyo Univ. of Agriculture and Technology, Tokyo 184, Japan

${ }^{(6)}$ Department of Physics, University of Tokyo, Tokyo 113, Japan

(7) Department of Physics, Osaka City University, Osaka 558, Japan

${ }^{(8)}$ Department of Physics, Purdue University, West Lafayette, IN 47907, USA

(9) Institute for Nuclear Study, University of Tokyo, Tanashi, Tokyo 188, Japan

${ }^{(10)}$ The Graduate School of Science and Technology, Kobe University, Kobe 657, Japan

(11) Faculty of Liberal Arts, Tezukayama University, Nara 631, Japan

(January 1, 2018)

\begin{abstract}
We have observed highly virtual $\left(Q^{2}>1.05 \mathrm{GeV}^{2}\right)$ photon-photon collisions to hadronic final states at $\sqrt{s_{e^{+} e^{-}}}=58 \mathrm{GeV}$. The integrated luminosity of the data sample was $241 \mathrm{pb}^{-1}$. Both scattered beam-electrons and scattered beam-positrons were detected using low-angle calorimeters (i.e., both photons were highly virtual, "double-tag"); we obtained 115 hadronic events with an estimated background of $10.2 \pm 1.1$. The cross section obtained was $4.11 \pm 0.66 \mathrm{pb}$ in the $2<W_{\gamma \gamma}<25 \mathrm{GeV}$ and $Q_{\gamma, \min }^{2}>2 \mathrm{GeV}$ region, while the lowest order quark-parton model predicted 3.00pb.

13.65.+i, 12.38.Qk

keywords: photon-photon collision, hadronic final state, double-tag, virtual photon, total cross section.
\end{abstract}

Typeset using REVTEX 
A measurement of the total hadronic cross section in highly virtual photon-photon collisions ( $e^{+} e^{-} \rightarrow e^{+} e^{-}$hadrons) is reported for center-of-mass energy, $W$, between 2 and 25 $\mathrm{GeV}$. In this experiment both the scattered $e^{+}$and $e^{-}$, refered as tags, were detected. Such a technique is called "double-tag" [1]. The range of photon four-momentum squared, $q^{2}$, for this experiment was -1.05 to $-37 \mathrm{GeV}^{2}$. $q^{2}$ 's and $W$ were detected on an event-by-event basis directly from the tags. Theoretical calculation of such events in which two large scales of virtuality exist, does not rely on any high $P_{T}$ selection in the hadronic final state. The measurement thus amounts to a first measurement of a well defined total hadronic cross section in which there could be minimal sensitivity to QCD cut-offs.

Examples of Feynman diagrams which contribute to $\gamma \gamma$ collisions are shown in Figures 1 1 (a)-(e). Figure 1 (a) is called multi-peripheral or "direct process" which is expected to be dominant in these events [2]. Figures 1 (b), (c), and (d) are examples of a bremsstrahlung, conversion, and annihilation diagram. Figure 1 (e) is an example of "resolved photon process" which is considered to be a smaller contribution in highly virtual photon-photon collisions [3].

The statistics and virtualities of the photons of the experimental data are the highest compared with previous experiments [4].

The integrated luminosity of the event sample is $241 \mathrm{pb}^{-1}$ at $\sqrt{s}=58 \mathrm{GeV}$. The details concerning the TOPAZ detector at TRISTAN can be found in reference [5].

To select virtual photon-photon collision events, we used a pair of low-angle calorimeters (FCL) made of Bismuth Germanate crystals (BGO) [6]. This covered the polar-angle region of 3.2 to 12 degrees with respect to the beam axis. The energy resolution was measured to be $5 \%$ for Bhabha electrons, and 3\% for $4-\mathrm{GeV}$ electron beams. We required that both of the FCL's (electron and positron sides) had single clusters of energies greater than $0.4 E_{b}$ where $E_{b}$ is the beam energy. This cut was determined in order to reduce contributions from the resolved-photon process [7] and initial (final) state radiation in various processes. It also helped us to reject any background from spent electrons in the accelerator beams. Under these conditions the virtuality of the photon $\left(Q^{2}=-q^{2}\right)$ was ensured to be greater 
than $1.05 \mathrm{GeV}^{2}$.

The trigger system for the TOPAZ detector consisted of neutral and charged-track triggers. The conditions for the neutral triggers were as follows: (a) The total energy deposited in a barrel calorimeter (BCL, made of lead-glass) or an endcap calorimeter (ECL, made of $\mathrm{Pb}$-streamer-tube sandwiches) must be greater than 2 or 4 (63\% of the events were taken with a $2-\mathrm{GeV}$ threshold of the BCL, and the others with a 4-GeV threshold.) and $10 \mathrm{GeV}$, respectively; (b) The BCL was segmented into three parts and the ECL into 4 parts. For energy deposits in these parts, $1 \mathrm{GeV}$ (for BCL) and $4 \mathrm{GeV}$ (for ECL) thresholds were set. Any two hits caused trigger signals. The charged-track trigger required at least two tracks from the origin. The $P_{T}$ of each track was required to be greater than $0.3 \sim 0.7 \mathrm{GeV}$ (run dependent), and the opening angle between one of the pairs must be greater than 45 90 degrees. The cut values depended on the beam conditions; their variation throughout the data taking were taken into account in Monte-Carlo simulations. The details can be found in reference [8].

We used a time-projection-chamber (TPC), the BCL, and the ECL in event selection. Detailed analyses of these detectors can be found in reference [9].

We required at least three charged tracks from the origin with a $P_{T}$ greater than 0.15 $\mathrm{GeV}$. The invariant mass $\left(W_{V I S}\right)$ of the visible particles (in the TPC and BCL) had to be greater than $2 \mathrm{GeV}$. Here, the energy threshold of the BCL neutral clusters had to be greater than $100 \mathrm{MeV}$. The visible energy in this region had to be less than $25 \mathrm{GeV}$.

In order to reduce the backgrounds from annihilation events which were produced at low angle, we required that the energy flow into the ECL be less than that into the BCL.

The material thickness in front of the TPC was $\sim 0.3$ radiation length. Therefore, we had to pay special attention to $e^{+} e^{-} \rightarrow e^{+} e^{-} e^{+} e^{-}(\gamma)$. Using $\mathrm{dE} / \mathrm{dx}$ measurements from the TPC we identified electron tracks in the events. To reject this process, we required that an event must contain at least two non-electron tracks.

Finally, we obtained a total of 115 events. We investigated three sources of background: (a) beam-gas and accidental coincidences by spent electrons, (b) $e^{+} e^{-} \rightarrow q \bar{q}(n \gamma)$, and (c) 
$e^{+} e^{-} \rightarrow e^{+} e^{-} l^{+} l^{-}(n \gamma)$ where $l$ is a lepton.

- (a) Using the Bhabha-scattering events in the central region of the detector, as well as random triggered events, we estimated the background level of the accidental hits in the FCL. The accidental rate was measured to be less than $0.1 \%$. The contamination of the beam-gas scattering was estimated by using off-vertex events in the experimental sample. It was estimated to be $1.0 \pm 0.7$ events.

- (b) The KORALZ generator was used to simulate the initial (ISR) and final (FSR) state radiation with multiple $\gamma^{\prime}$ ' [10,11]. Here, the maximum energy $\left(k_{\max }\right)$ of the radiated photon was set at $0.97 E_{b}$ for b-quark events and $0.99 E_{b}$ for the other quarks. The next-to-leading order $\left(\alpha_{s}\right)$ correction and hadronization was carried out using JETSET 6.3 [11, 12]. The contamination of this process was estimated to be $2.3 \pm 0.7$ events.

- (c) The FERMISV generator was used to simulate this process [13]. This generator could also simulate ISR and FSR. $k_{\max }$ was set at $0.8 E_{b}$. The contamination was estimated to be $6.9 \pm 0.6$ events; $\tau$-pair events dominantly contributed ( $6.0 \pm 0.5$ events).

In total, the background contamination in this sample was estimated to be $10.2 \pm 1.1$ events. We have thus obtained a clean sample of direct photon-photon collision events.

The average of smaller and larger $Q^{2}$ 's of two photons of this event sample were 5.1 and $12.3 \mathrm{GeV}^{2}$. Both are equally highly virtual. We derived the differential cross sections of $e^{+} e^{-} \rightarrow e^{+} e^{-}$hadrons with respect to $W_{\gamma \gamma}$ at several $Q_{\gamma, \min }^{2}$ cuts, where $Q_{\gamma, \min }^{2}$ was a minimum of two $Q_{\gamma}^{2}$ detected by the forward and backward FCL counters. The cross section such as $\sigma_{\gamma \gamma \rightarrow \text { hadrons }}$ or photon structure function would be a future subject in which we need more statistics and works. We used the FERMISV code for deriving the acceptance correction factors with our detector simulation codes. We again set $k_{\max }=0.8 E_{b}$ for ISR and FSR in FERMISV. We restricted events with all four fermions in the final state having polar angles greater than 2.5 degrees from the beam axis. The minimum invariant masses 
for hadronic system were set to $2 \mathrm{GeV}$ for light quark events, $2 m_{D}$ for c, and $2 m_{B}$ for b, respectively. The cosines of scattered electrons (positrons) were required to be within $0.9762<|\cos \theta|<0.9986$. Quark masses used were $0.35 \mathrm{GeV}$ for $\mathrm{u}, \mathrm{d}, 0.5 \mathrm{GeV}$ for s, 1.35 $\mathrm{GeV}$ for $\mathrm{c}$, and $4.5 \mathrm{GeV}$ for b. The hadronization process was simulated using JETSET 6.3 string fragmentation [12,14]. The obtained cross sections are shown in Table — for various kinematic ranges. Also shown are the ratios between the experimental and theoretical cross sections and the T-ratios which are the ratio between $\sigma_{\text {exp }}\left(e^{+} e^{-} \rightarrow e^{+} e^{-} q \bar{q}\right) / \sigma_{\text {threory }}\left(e^{+} e^{-} \rightarrow\right.$ $e^{+} e^{-} \mu^{+} \mu^{-}$), where only the multi-peripheral diagram is taken into account in calculating $\sigma_{\text {theory }}$. Radiative corrections were carried out in deriving these values. The cross section obtained was $4.11 \pm 0.66 \mathrm{pb}$ in the $2<W_{\gamma \gamma}<25 \mathrm{GeV}$ and $Q_{\gamma, \min }^{2}>2 \mathrm{GeV}$ region, while the lowest order (LO) theory predicted by FEMISV is 3.00pb. Figure 2 shows the ratios between the experimental and LO-theoretical cross sections. Observed distributions such as $W_{V I S}$ and number of charged tracks were consistent with the Monte-Carlo prediction within statistical errors except for overall normalizations. On the other hand, the experimental data agreed with the theoretical predictions at high $Q_{\gamma}^{2}$, including overall normalization factors. The errors shown are both statistical and systematic. We consider the systematic errors below.

The systematic errors were estimated separately concerning the FCL, trigger, and event selection.

- FCL: We changed the energy threshold in the clustering algorithm, as well as that in electron tagging. The dependence of the acceptance was compared with a Monte-Carlo simulation. Also, background contamination was studied by comparing it with Bhabha and random-triggered events. The systematic error due to the FCL was estimated to be $5.2 \%$.

- Trigger: We added noise hits in the tracking detector to the Monte-Carlo simulations. We also studied the acceptance dependence on the energy thresholds of the neutral triggers. The error was estimated to be $6.0 \%$ based on the ambiguity in the hardware 
settings $(10 \%)$.

- Event selection: We changed the cut values and derived the cross sections. The differences were taken into account as errors $(4.9 \%)$.

- Hadronization: We used JETSET 6.3 for hadronization of $q \bar{q}$ system [12]. We changes parameters of string fragmentation $a$ and $\sigma_{q}$ in the ranges $0.4-0.7 \mathrm{GeV}^{-2}$ and $0.35-0.45$ $\mathrm{GeV}$, respectively. The acceptance differences were taken into account as systematic errors. Their bin-to-bin average was $8.9 \%$.

An error of integrated luminosity was estimated to be $4 \%$. The errors except for luminosity and trigger were derived by a bin-by-bin bases. For example a luminosity error is common for all bins, i.e., correlates positively. The correlated systematic error was estimated to be $7.2 \%$. The average bin-by-bin systematic errors was estimated to be $13.5 \%$. The first errors quoted in Table $\mathbb{1}$ are statistical and the second ones are systematic. The errors in Figure 2 are quadric sums of these errors.

The accuracy of the FCL energy measurements is important for determining $W_{\gamma \gamma}$. The FCL energy was originally calibrated at $4 \mathrm{GeV}$ using AR electron beams at KEK and at $29 \mathrm{GeV}$ by the Bhabha-scattering electrons. In order to check the other energy region, we studied radiative Bhabha events. We selected acoplanar Bhabha events where the radiated $\gamma$ direction was consistent with the FCL fiducial volume. The agreement between the BCLpredicted and the FCL-measured energies was as expected from our Monte-Carlo simulation. The Monte-Carlo simulation predicted that the $W_{\gamma \gamma}$ resolution would be almost constant $(\sim 3 \mathrm{GeV})$ in the observed energy region $\left(2<W_{\gamma \gamma}<25 \mathrm{GeV}\right)$. This was better than the $W_{V I S}$ determined from the TPC and BCL.

In conclusion, we have observed highly virtual $\left(Q^{2}>1.05 \mathrm{GeV}^{2}\right)$ photon-photon collisions to hadronic final states at $\sqrt{s_{e^{+} e^{-}}}=58 \mathrm{GeV}$. The integrated luminosity of the data sample was $241 \mathrm{pb}^{-1}$. The beam electrons (positrons) were tagged by low-angle calorimeters; we obtained $97.3 \pm 10.3$ events in the $2<W_{\gamma \gamma}<25 \mathrm{GeV}$ and $Q_{\gamma, \text { min }}^{2}>2 \mathrm{GeV}$ region, the Monte-Carlo with LO theory predicted $65.6 \pm 2.2$ events where the errors are statistical. In 
this new kinematic region the contribution of the LO $q \bar{q}$ diagram is expected to dominate. With systematic errors included, the ratio between experimental and theoretical data becomes $1.48 \pm 0.26$. The cross sections were measured to be $4.11 \pm 0.66 \mathrm{pb}$ in the region where $Q^{2}>2 \mathrm{GeV}^{2}$ and $2<W_{\gamma \gamma}<25 \mathrm{GeV}$.

We thank Drs. H. Terazawa, M. Kobayashi, K. Hagiwara, and T. Izubuchi for helpful discussions. We also thank the TRISTAN accelerator staff for the successful operation of TRISTAN. The authors appreciate all of the engineers and technicians at KEK as well as those of the collaborating institutions: H. Inoue, N. Kimura, K. Shiino, M. Tanaka, K. Tsukada, N. Ujiie, and H. Yamaoka. 


\section{REFERENCES}

[1] H. Terazawa, Phys. Rev. D 5 (1972) 2259; T. Uematsu and T. F. Walsh, Nucl. Phys. B 199 (1982) 93; G. Rossi, Phys. Rev. D 29 (1984) 852; F. M. Borzumati and G. A. Schuler, Z. Phys. C 58 (1993) 139.

[2] S. J. Brodsky, T. Kinoshita, and H. Terazawa, Phys. Rev. D4 (1971) 1532.

[3] S. J. Brodsky, T. A. DeGrand, J. F. Gunion, and J. H. Weis, Phys. Rev. Lett. 41 (1978) 672; Phys. Rev. D 19 (1979) 1418; H. Terazawa, J. Phys. Soc. Jpn. 47 (1979) 355; K. Kajantie and K. Grassie, Nucl. Phys. B159 (1979) 528.

[4] Ch. Berger et al., Phys. Lett. B142 (1984) 119; B. Bintinger et al., Phys. Rev. Lett. 54 (1985) 763; H. Aihara et al., Phys. Rev. D41 (1990) 2667; S. E. Baru et al., Z. Phys. C53 (1992) 219.

[5] T. Kamae et al., Nucl. Instrum. Meth. A252 (1986) 423; S. Kawabata et al., Nucl. Instrum. Meth. A270 (1988) 11; J. Fujimoto et al., Nucl. Instrum. Meth. A256 (1987) 449; A. Yamamoto et al., Jpn. J. Appl. Phys. Lett. 55 (1986) L440.

[6] H. Hayashii et al., Nucl. Instrum. Meth. A316 (1992) 202.

[7] R. Enomoto et al., Phys. Lett. B341 (1994) 238.

[8] R. Enomoto et al., Nucl. Instrum. Meth. A269 (1988) 507; R. Enomoto, K. Tsukada, N. Ujiie, and A. Shirahashi, IEEE Trans. Nucl. Sci. NS-35 (1988) 419; T. Tsukamoto, M. Yamauchi, and R. Enomoto, Nucl. Instrum. Meth. A297 (1990) 148.

[9] M. Iwasaki, E. Nakano, and R. Enomoto, KEK preprint 94-180, to be published in Nucl. Instrum. Meth. A.

[10] S. Jodash and B. F. L. Ward, Comput. Phys. Cumm. 66 (1991) 276.

[11] K. Miyabayashi et al., Phys. Lett. B347 (1995) 171. 
[12] T. Sjöstrand, Comput. Phys. Commun. 39 (1986) 347; T. Sjöstrand and M. Bengtsson, Comput. Phys. Commun. 43 (1987) 367.

[13] J. Hilgart, R. Kleiss, and F. L. Diberder, Comput. Phys. Commun. 75 (1993) 191.

[14] R. Enomoto et al., Phys. Lett. B328 (1994) 535. 


\section{TABLES}

\begin{tabular}{lccccc}
\hline \hline \multicolumn{2}{c}{ Kinematic Region } & Cross section & ratio(Exp/Th) & T-ratio \\
$Q_{\gamma, \text { min }}^{2}$ & $W_{\gamma}$ range & $\sigma_{\text {hadron }}^{\text {exp }}$ & $\sigma_{\text {hadron }}^{\text {theory }}$ & $\sigma_{\text {hadron }}^{\text {exp }} / \sigma_{\text {hadron }}^{\text {theory }}$ & $\sigma_{\text {hadron }}^{\text {exp }} / \sigma_{\mu^{+} \mu^{-}}^{\text {theory }}$ \\
$\left(\mathrm{GeV}^{2}\right)$ & $(\mathrm{GeV})$ & $(\mathrm{pb})$ & $(\mathrm{pb})$ & & \\
\hline $2.0(8.5)$ & $2.0-6.0(4.6)$ & $2.41 \pm 0.48 \pm 0.33$ & 1.81 & $1.33 \pm 0.26 \pm 0.17$ & $1.17 \pm 0.23 \pm 14$ \\
$2.0(10.7)$ & $6.0-9.5(7.8)$ & $0.89 \pm 0.20 \pm 0.19$ & 0.71 & $1.25 \pm 0.28 \pm 0.26$ & $1.36 \pm 0.30 \pm 0.28$ \\
$2.0(8.2)$ & $9.5-13.5(11.4)$ & $0.51 \pm 0.10 \pm 0.09$ & 0.30 & $1.69 \pm 0.32 \pm 0.32$ & $1.79 \pm 0.34 \pm 0.36$ \\
$2.0(8.6)$ & $13.5-25.0(18.6)$ & $0.295 \pm 0.056 \pm 0.060$ & 0.186 & $1.59 \pm 0.30 \pm 0.33$ & $1.85 \pm 0.35 \pm 0.39$ \\
$3.5(9.7)$ & $2.0-7.0(5.2)$ & $1.03 \pm 0.23 \pm 0.15$ & 1.00 & $1.04 \pm 0.23 \pm 0.15$ & $1.01 \pm 0.22 \pm 0.15$ \\
$3.5(10.5)$ & $7.0-12.0(9.4)$ & $0.47 \pm 0.10 \pm 0.09$ & 0.35 & $1.34 \pm 0.28 \pm 0.25$ & $1.49 \pm 0.31 \pm 0.28$ \\
$3.5(10.8)$ & $12.0-25.0(16.2)$ & $0.185 \pm 0.039 \pm 0.039$ & 0.143 & $1.29 \pm 0.27 \pm 0.28$ & $1.45 \pm 0.30 \pm 0.32$ \\
$5.0(12.9)$ & $2.0-9.0(6.4)$ & $0.62 \pm 0.12 \pm 0.15$ & 0.67 & $0.93 \pm 0.18 \pm 0.22$ & $0.99 \pm 0.19 \pm 0.23$ \\
$5.0(12.3)$ & $9.0-25.0(14.2)$ & $0.179 \pm 0.030 \pm 0.051$ & 0.184 & $0.97 \pm 0.16 \pm 0.27$ & $1.12 \pm 0.19 \pm 0.31$ \\
\hline \hline
\end{tabular}

TABLE I. Hadronic cross sections in various kinematic ranges. The first errors are statistical and the second ones are systematic errors as described in the text. The terms shown in this table are specified in the text. The values in parentheses in the first and second columns are event average of the kinematic variables. 


\section{FIGURES}

FIG. 1. Examples of Feynman diagrams which contribute to $\gamma \gamma$ collisions; (a) multi-peripheral, (b) bremsstrahlung, (c) conversion, (d) annihilation, and (e) "resolved photon process".

FIG. 2. Ratios of experimental and theoretical cross sections in various kinematic regions; (a)

$Q_{\gamma, \text { min }}^{2}>2 \mathrm{GeV}^{2}$, (b) $Q_{\gamma, \text { min }}^{2}>3.5 \mathrm{GeV}^{2}$, and (c) $Q_{\gamma, \text { min }}^{2}>5 \mathrm{GeV}^{2}$. The $Q_{\gamma, \text { min }}^{2}$ is specified in the text. 
FIGURE 1, PHYS. LETT. B., R. ENOMOTO ET AL.

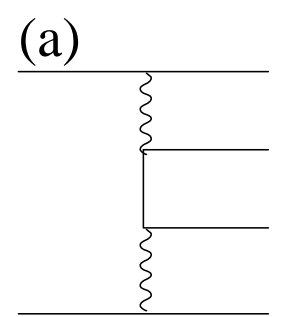

(b)
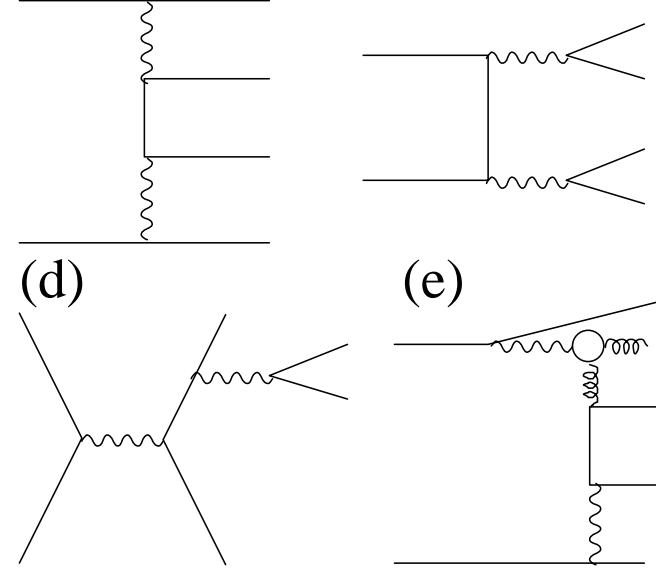

(c)

(e)

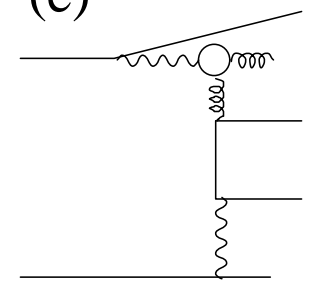


FIGURE 2, PHYS. LETT. B., R. ENOMOTO ET AL.

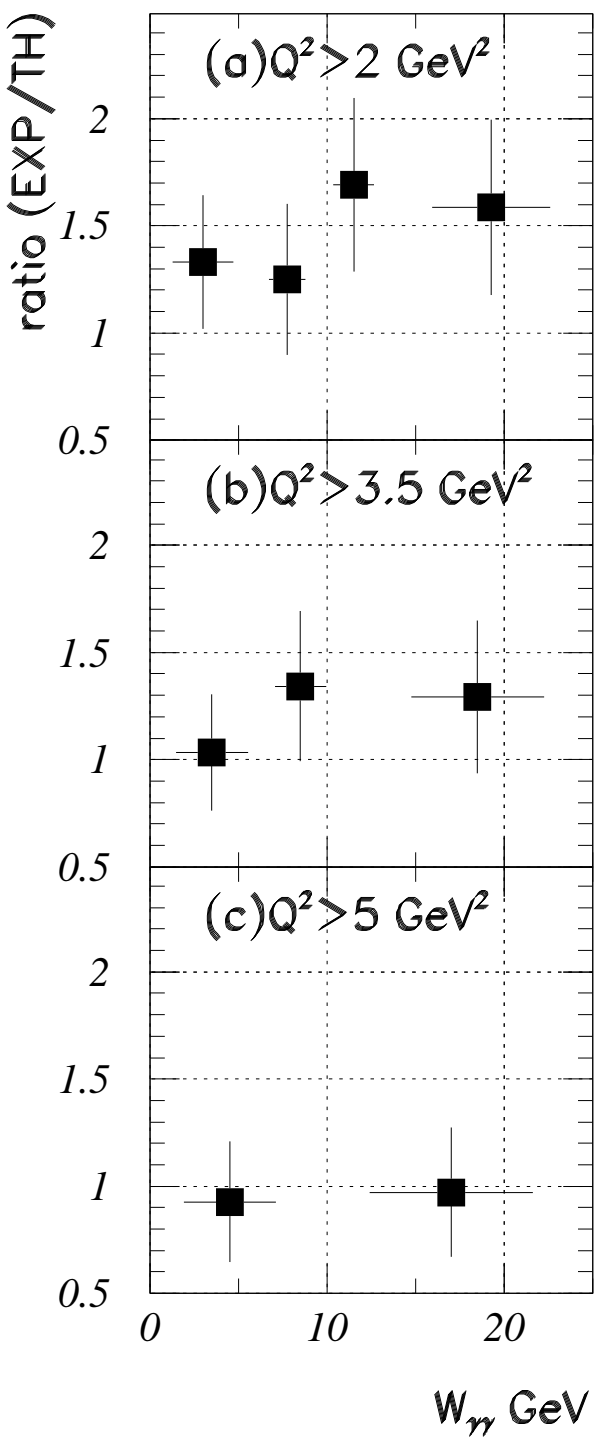

